Pacific Journal of Mathematic 


\section{THE SILOV BOUNDARY FOR A LATTICE-ORDERED SEMIGROUP}

\section{J. C. TAYLOR}

Let $X$ be a compact Hausdorff space and let $S$ be a point separating collection of $\overline{\mathbf{R}}^{+}$-valued lower semicontinuous functions on $X$ which is closed under addition. Assume that $S$ is a lower semi-lattice with respect to the partial order $\leqq$ (where $f \leqq g$ if $g=f+h$, for some $h \in S$ ). Further, assume $S$ contains all the nonnegative constant functions $\lambda$ and is such that $\lambda \leqq f$ implies $\lambda \leqq f$ (where $\lambda \leqq f$ if $\lambda \leqq f(x)$ for all $x \in X$ ). Then, the Silov boundary of $S$ is precisely $\{x \mid(f \wedge g)(x)=$ min $\{f(x), g(x)\} \forall f, g \in S\}$ if, in addition, for all $f, g$, and $h \in S$ we have $f+(g \wedge h)=(f+g) \wedge(f+h)$.

This theorem ${ }^{1}$ extends a result due to $\mathrm{H}$. Bauer [1]. He showed that if $H \subseteq C(X, \mathbf{R})$ is a linear subspace which contains the constant functions, separates the points of $X$, and is a lattice with respect to the partial order $\leqq$, then the Šilov boundary of $H$ is precisely $\{x \mid$ $(f \wedge g)(x)=\min \{f(x), g(x)\} \forall f, g \in H\}$. This result can be obtained from the above theorem by applying it to the semigroup $H^{+}$of nonnegative functions in $H$.

The analogous theorem for upper semi-lattices is false. If, however, $S$ is a lattice with respect to $\leqq$ which satisfies the hypotheses of the theorem, then $\partial S \subseteq\{x \mid(f \vee g)(x)=\max \{f(x), g(x)\} \quad \forall f, g \in S\}$ and this inclusion can be proper.

2. Basic assumptions. Let $X$ be a compact Hausdorff space and let $S$ be a set of $\overline{\boldsymbol{R}}^{+}$- valued lower semicontinuous functions on $X$ which is closed under addition and separates the points of $X$. A closed set $B \subseteq X$ is called a boundary for $S$ if each function in $S$ attains its minimum on $B$. Bauer [1] has shown that there exists a boundary, denoted by $\partial S$ and called the Silov boundary of $S$, which is a subset of every boundary.

Two partial orders $\leqq$ and $\leqq$ can be defined on $S$ which are compatible with addition. Set $f \leqq g$ if $f(x) \leqq g(x)$ for all $x \in X$ and set $f \leqq g$ if $g=f+h$, for some $h \in S$. Then, clearly, $f \leqq g$ implies $f \leqq g$.

Assume that $S$ is a lower semi-lattice with respect to $\leqq$ (i.e. if $f$ and $g$ are in $S$ their meet $f \wedge g$ exists in $S$ ). Let $A=\{x \in X \mid \forall f$,

1 The author wishes to thank the referee for extending this theorem from continuous functions to lower semicontinuous functions, the crucial step being the arguement for Proposition 1, and for shortening the arguement of Proposition 2. 
$g \in S,(f \wedge g)(x)=\min \{f(x), g(x)\}\}$. Then, as the following, examples show, not much can be said about the relationship of $A$ and $\partial S$.

Example 1 (due to E.J. Barbeau). Let $X_{1}=\{1,2,3\}$ and let $S_{1}$ be the set of positive real-valued functions $f$ on $X$ with $f(1) \leqq 1 / 2$ $(f(2)+f(3))$. Then $S$ is a point separating subcone of $C(X, \mathbf{R})$ which contains the constants and is a lattice with respect to the partial order $\leqq$. In fact, for $f, g \in S_{1},(f \vee g)(i)=\max \{f(i), g(i)\},(f \wedge g)$ $(j)=\min \{f(j), g(j)\}$ if $j=2$ or 3 , and $(f \wedge g)(1)=1 / 2[(f \wedge g)(2)+$ $(f \wedge g)(3)]$. The set $A=\{2,3\}$ and $\partial S_{1}=X_{1}$.

ExAmple 2. Let $X_{2}=\{4,5\}$ and let $S_{2}$ be the set of positive realvalued functions for $X$ with $f(4) \leqq f(5)$. Then $\partial S_{2}=\{4\}$ and $A=X_{2}$.

ExAmple 3. Let $X=X_{1} \cup X_{2}$ and let $S$ be the set of functions $f$ with $f \mid X_{i} \in S_{i}$. Then $A=\{2,3,4,5\}$ and $\partial S=\{1,2,3,4\}$.

Assume now that $S$ is a lower semi-lattice with respect to $\leqq$, Define $A$, as before, to be $\{x \mid(f \wedge g)(x)=\min \{f(x), g(x)\} \quad \forall f, g \in S\}$, where $f \wedge g$ now denotes meet with respect to $\leqq$.

\section{Lemma. $A$ is closed.}

Proof. If $x_{0} \notin A$ then, for some $f$ and $g \in S$, there is $\lambda>0$ with $\min (f, g)\left(x_{0}\right)>\lambda>(f \wedge g)\left(x_{0}\right)$. Set $f_{1}=f \wedge \lambda$ and $g_{1}=g \wedge \lambda$ Then, since $f_{1} \wedge g_{1}=(f \wedge g) \wedge \lambda$, one of the inequalities $\min (f, \lambda)\left(x_{0}\right) \geqq f_{1}$ $\left(x_{0}\right), \min (g, \lambda)\left(x_{0}\right) \geqq g_{1}\left(x_{0}\right)$, and $\min \left(f_{1}, g_{1}\right)\left(x_{0}\right) \geqq\left(f_{1} \wedge g_{1}\right)\left(x_{0}\right)$ is strict.

Consequently, if $x_{0} \notin A$ there exist $f, g \in S$ with $g$ real-valued on $X$ and $\min (f, g)\left(x_{0}\right)>(f \wedge g)\left(x_{0}\right)$. Now $f=f \wedge g+h$ and $g=f \wedge$ $g+k$, so $\min (f, g)=f \wedge g+\min (h, k)$. Since $\min (h, k)\left(x_{0}\right)>0$, $\min (h, k)$ is lower semi-continuous, and $g$ is real-valued, a neighbourhood of $x_{0}$ is disjoint from $A$.

Proposition 1. Let $x_{0} \in A$ and let $U$ be an open neighbourhood of $x_{0}$. If $S$ contains all the nonnegative constant functions there exist $\varepsilon>0$ and $f \in S$ with (1) $f\left(x_{0}\right)=0$ and (2) $\{x \mid f(x)<\varepsilon\} \subseteq U$.

Proof $^{2}$. Assume the proposition to be false. Then as $f \in S$, with $f\left(x_{0}\right)=0$, and $\varepsilon>0$ vary, the closed sets of the form $\{x \notin U \mid f(x)$ $\geqq \varepsilon\}$ define a filter base. Hence there is a point $x_{1} \notin U$ such that if $f \in S$ and $f\left(x_{0}\right)=0$, then $f\left(x_{1}\right)=0$.

For $g \in S$ if $\lambda=g\left(x_{0}\right)<+\infty$, then $(g \wedge \lambda)\left(x_{0}\right)=\lambda$. Now there

${ }^{2}$ This arguement, based on a technique of Loeb and Walsh in [3] is due to the referee. 
exist functions $h$ and $k \in S$ with $g \wedge \lambda+h=g$ and $g \wedge \lambda+k=\lambda$. Since $h\left(x_{0}\right)=k\left(x_{0}\right)=0$ it follows that $g\left(x_{1}\right)=(g \wedge \lambda)\left(x_{1}\right)=\lambda=g\left(x_{0}\right)$. If $g\left(x_{0}\right)=+\infty$, then $n=(g \wedge n)\left(x_{0}\right)=(g \wedge n)\left(x_{1}\right) \leqq g\left(x_{1}\right)$ and so $g\left(x_{1}\right)$ $=+\infty$. This contradicts the fact that $S$ separates points.

COROLLARY. If $S$ is a lower semi-lattice with respect to $\leqq$ which contains all the nonnegative constant functions, then $A \leqq \partial S$.

3. The main theorem. From now on $S$ is assumed to have the following properties:

(a) $S$ contains all the nonnegative constant functions $\lambda$.

(b) $\lambda \leqq f$ implies $\lambda \leqq f$; and

(c) for all $f, g, h \in S, f+(g \wedge h)=(f+g) \wedge(f+h)$.

Proposition 2. Let $I \subseteq S$ be maximal with respect to the following properties:

(1) $f, g \in I \Rightarrow f+g \in I$

(2) $g \leqq f$ and $f \in I \Longrightarrow g \in I$

(3) $1 \notin I$.

Then there is a point $x_{0} \in A$ with $I=\left\{f \in S \mid f\left(x_{0}\right)=0\right\}$.

Assuming this proposition the main theorem of this note is quickly proved.

THEOREM. Let $X$ be compact Hausdorff and let $S$ be a pointseparating collection of lower semi-continuous functions $f: X \rightarrow[0,+$ $\infty]$, Assume that $S$ is closed under addition and is a lower semilattice with respect to the partial order $\leqq$ (where $f \leqq g$ if $g=f+$ $h$, for some $h \in S$.)

The Silov boundary of $S$ coincides with $A=\{x \mid \forall f, g \in S,(f \wedge g)(x)$ $=\min \{f(x), g(x)\}\}$ if $S$ satisfies properties (a), (b) and (c).

In particular this is the case if $S$ satisfies (a), (b) and the cancellation law.

Proof. Since $A$ is a closed subset of $\partial S$ it suffices to show that each $f \in S$ with finite minimum $\alpha$ attains $\alpha$ on $A$. Let $M=\{x(f(x)=$ $\alpha\}$. Then, since $f \wedge \alpha=\alpha$ there exists a function $h \in S$ with $f=$ $\alpha+h$ which vanishes on $M$. Let $I_{0}$ be the set of functions in $S$ which vanish on $M$. Then $I_{0}$ satisfies conditions (1), (2) and (3) of Proposition 2. Since $I_{0}$ can be embeded in a set $I \subseteq S$ maximal with respect to these properties, it follows from Proposition 2 that $M \cap$ $A \neq \phi$.

ExAMPLE 4. Let $X=\{1,2,3\}$ and let $S$ be the semigroup of 
positive real-valued functions $f$ with $f(1) \leqq f(2)$ and $3 / 4 f(2)-1 / 4 f(1)$ $\geqq f(3)$. Then $S$ satisfies all the hypotheses of the theorem except (b). Here $A=\{1\}$ and $\partial S=\{1,3\}$.

4. Proof of proposition 2. Let $B=\{x \mid f \in I \Rightarrow f(x)=0\}$. Then, since $I$ satisfies (1), (2) and (3), $B \neq \varnothing$ and furthermore, if $\varnothing \neq K \subseteq \mathcal{E} B$ is compact there exists $h \in I$ with $\min _{K} h>0$. The maximality of $I$ implies that if $h \in S$ vanishes at a point of $B$, then $h \in I$.

Define $\psi: S \rightarrow \mathbf{R}^{+}$by setting $\psi(f)=\min _{B} f$. Then $\psi$ has the following properties:

( i ) $\psi(f)=\sup \{\lambda \mid$ for some $h \in I, \lambda \leqq f+h\}$

(ii) $\psi(f)=0 \Leftrightarrow f \in I$

(iii) $\psi(f \wedge \psi(f))=\psi(f)$

(iv) $f \in S \Rightarrow f|B=\psi(f)| B$.

(i ) Let $\lambda<\psi(f)$. Then $K=\{x \mid f(x) \leqq \lambda\}$ is compact and disjoint from $B$. If $K \neq \varnothing$, there exists $h \in I$ with $\min _{K} h \geqq \lambda$ and so $\lambda$ $\leqq f+h$. If $\lambda \leqq f+h$ then $\lambda \leqq \psi(f)$ as $h$ vanishes on $B$.

( ii ) Clear.

(iii) $\psi(f \wedge \psi(f)) \leqq \psi(f)$ anyway. Let $\lambda<\psi(f)$ and let $h \in I$ with $\lambda \leqq f+h$. Now $\lambda \leqq \psi(f)+h$ implies by (c) that $\lambda \leqq f \wedge \psi(f)$ $+h$ and so $\lambda \leqq \psi(f \wedge \psi(f))$.

(iv) Assume $\psi(f)<+\infty$ as it is trivial if $\psi(f)=+\infty$. Then $f=f \wedge \psi(f)+g$ for some $g \in S$. Now $\psi(f) \geqq \psi(f \wedge \psi(f))+\psi(g)=$ $\psi(f)+\psi(g)$ and so $\psi(g)=0$. Consequently, $f|B=f \wedge \psi(f)| B$ and hence $f|B=\psi(f)| B$.

Property (iv) implies that $B=\left\{x_{0}\right\}$ for some $x_{0} \in X$. It remains to show that $x_{0} \in A$. Let $f, g \in S$ and let $\lambda=f\left(x_{0}\right) \wedge g\left(x_{0}\right)$. If $\lambda \leqq$ $(f \wedge g)\left(x_{0}\right)$ then $\lambda=(f \wedge g)\left(x_{0}\right)$ and so $x_{0} \in A$.

Let $\alpha<\lambda$. Then there exist functions $h$ and $k$ in $I$ with $\alpha \leqq$ $f+h$ and $\alpha \leqq g+k$. Property (c) implies that $\alpha \leqq \alpha+(h \wedge k)=$ $(\alpha+h) \wedge(\alpha+k) \leqq(f+h+k) \wedge(g+h+k)=(f \wedge g)+(h+k)$. Hence, $\alpha \leqq \psi(f \wedge g)=(f \wedge g)\left(x_{0}\right)$.

Remarks. This arguement, due to the referee, is a shortened version of an argument of the author which showed that $A$ could be identified with the additive functions $\dot{\psi}: S \rightarrow \overline{\boldsymbol{R}}^{+}$that preserve finite meets and for which $\psi(\lambda)=\lambda$ if $\lambda$ is a constant. (c.f. Bauer [1]).

5. Upper semi-lattices. Examples 1,2 and 3 show that when $S$ is an upper semi-lattice with respect to $\leqq$ there is no particular 
relationship between $\partial S$ and $B=\{x \mid(f \vee g)(x)=\max \{f(x), g(x)\} \forall f$, $g \in S\}$.

Assume that $S$ is an upper semi-lattice with respect to $\leqq$. Then, if $S$ is also a lower semi-lattice with respect to $\leqq$ and if $S$ satisfies the hypotheses of the theorem, $\partial S \subseteq B$ whenever the identity $f+g=$ $f \vee g+f \wedge g$ holds for all $f, g \in S$ (for example, this is the case if $f+(g \vee h)=(f+g) \vee(f+h)$ for all $f, g h \in S)$. However, as the following example shows, $\partial S$ can be distinct from $B$.

ExAmple 5. Let $X=\{1,2\}$ and let $S$ be the set of nonnegative functions $f$ with $f(1)$ finite and $f(1)=f(2)$ or $f(2)=+\infty$. Then $S$ is a lower semi-lattice. Here $\partial S=A=\{1\}$. However, $B=X$ since for $f, g \in S \max (f, g)=f \vee g$.

If $S$ is an upper semi-lattice but not a lattice with respect to $\leqq$ then $B$ can be a proper subset of $\partial S$ as shown by the next example.

Example 6 . Let $X=\{1,2,3\}$ and let $S$ be the set of nonnegative real-valued functions $f$ with $f(3) \geqq 4 / 3 f(2)-1 / 3 f(1)$. Then $S$ contains the nonnegative constant functions $\lambda$ and $\lambda \leqq f$ implies $\lambda \leqq f$. $S$ is an upper semi-lattice.

Here, $B=\{1,2\}$ and $\partial S=X$. Consequently, $S$ is not a lower semi-lattice with respect to $\leqq$. For example, there is no function in $S$ which is the meet of $f=(1,1,1)$ and $g=(1,1 / 4,1 / 2)$.

Putting Examples 5 and 6 together, as was done before to obtain Example 3 from Examples 1 and 2, we see that for upper semi-lattices $S$, even those which satisfy hypotheses analogous to those of the theorem on lower semi-lattices, there is no particular relationship between $\partial S$ and $B$.

6. The case of a vector space. Let $X$ be compact and let $H \cong C(X, \boldsymbol{R})$ be a point-separating set of continuous functions $f: X \rightarrow \overline{\boldsymbol{R}}$ which is a lattice with respect to the partial order $f \leqq g$ if $f(x) \leqq$ $g(x)$ for all $x \in X$. Assume that $H$ has the following properties:

(1) if $f \in H$, then $R(f)=\{x|| f(x) \mid<+\infty\}$ is dense;

(2) if $f, g \in H$ there is a function $h \in H$ with $h(x)=f(x)+g(x)$, for $x \in R(f) \cap R(g)$;

(3) if $f \in H$ and $\lambda \in \boldsymbol{R}$ there is a function $k \in H$ with $k(x)=$ $\lambda f(x)$ for $x \in R(f)$; and

(4) $H$ contains the constant functions.

These properties imply that $H$ is a vector lattice. Denote by $S$ the positive cone $H^{+}$of $H$. Then $S$ is an additive semigroup with cancellation which is a lattice with respect to the partial order $f \leqq g$ if $g=f+h$, for some $h \in S$. Clearly, the hypotheses of the theorem are satisfied by $S$, and so the Silov boundary of $S$ is the set of points 
in $X$ such that $(f \wedge g)(x)=\min \{f(x), g(x)\}$ for all $f, g \in S$. Since $f+g=f \wedge g+f \wedge g$, this is the set of points at which the lattice operations hold pointwise.

Denote by $H^{*}$ the set $\{f \in H \mid$ for some $n, n \geqq|f|\}$. Then $H^{*}$ is the subvector lattice of $H$ consisting of bounded functions.

If $B \cong X$ is closed, it will be called a boundary for $H^{*}$ if (1) each function in $H^{*}$ attains its maximum on $B$, and (2) if $x \in B$ and $f(x)=f(y)$, for all $f \in H^{*}$, then $y \in B$. It is well known that $H^{*}$ has a unique minimal boundary if $H^{*}$ separates the points of $X$ (c.f. [1]). By passing to an identification space $Y$ of $X$ and taking inverse images of sets in $Y$, it then follows that $H^{*}$ has a unique minimal boundary in $X$. This set will be called the Šilov boundary of $H^{*}$.

Proposition 3. The Šlov boundary of $H^{+}$is the Šilov boundary of $H^{*}$.

Proof. Let $A$ denote the Šlov boundary of $H^{+}$. Then, $A$ is a boundary for $H^{*}$.

It is clear that each function in $H^{*}$ attains its maximum on $A$. It remains to show that if $x \in A$ and $f(x)=f(y)$, for all $f \in H^{*}$, then $y \in A$.

Assume $x \in A$ and $y \neq x$. Then, since $f=f^{+}-f^{-}$there exists $f \in H^{+}$with either $\lambda=f(x)>f(y) \geqq 0$ or $\lambda=f(x)<f(y) \leqq 0$. In the first case let $g=f \wedge \lambda$ and in the second case let $g=f \vee \lambda$. Then, since $x \in A, g(x)=\lambda$ and $g(y) \leqq f(y)$ or $g(y) \geqq f(y)$. Hence, in either case, $g(x) \neq g(y)$. Since $g \in H^{*}$, it follows that $A$ is a boundary for $H^{*}$.

The Silov boundary $A$ of $H^{\top}$ is the set of points in $X$ at which the lattice operations hold pointwise. Since $H^{*} \mid A$ separates the points of $A$ it is dense in $C(A, \mathrm{R})$. Hence, $A$ is the Silov boundary for $H^{*}$.

ExAMPLE 1. Let $H$ be the vector lattice of differences of positive harmonic functions on some open set $\Omega \subseteq \mathbf{R}^{n}$. Let $X$ be the compactification of $\Omega$ determined by $H$ ([2] p. 97). Viewing $H^{+}$as a cone of functions on $X$, it follows that the Silov boundary of $H^{+}$coincides with the Šlov boundary of $H^{*}$, which in this case consists of the bounded harmonic functions on $\Omega$. Further, if $H_{1}^{+}$denotes the functions on $\Omega$. Further, if $H_{1}^{+}$denotes the functions $f$ in $H^{+}$with $f \wedge$ $1 \neq 0$, the Šlov boundary of $H^{+}$coincides with that of $H^{+}$.

\section{REFERENCES}

1. H. Bauer, Silovscher Rand und Dirichletsches Problem, Ann. Inst. Fourier 11 (1961) $86-136$. 
2. C. Constaninescu and A. Cornea, Ideale Rander Riemannscher Flächen, SpringerVerlag, Berlin, 1963.

3. P.A. Loeb and B. Walsh, A maximal regular boundary for solutions of elliptic differential equations (to appear)

Received September 15, 1966.

MCGILL University 



\section{PACIFIC JOURNAL OF MATHEMATICS}

\section{EDITORS}

H. ROYDEN

Stanford University

Stanford, California

\section{J. P. Jans}

University of Washington

Seattle, Washington 98105

\section{J. DUGUNDJI}

Department of Mathematics University of Southern California Los Angeles, California 90007

\section{RICHARD ARENS}

University of California

Los Angeles, California 90024

\section{ASSOCIATE EDITORS}

E. F. BECKENBACH

B. H. NEUManN

F. WOLF

K. YosIDA

\section{SUPPORTING INSTITUTIONS}

UNIVERSITY OF BRITISH COLUMBIA CALIFORNIA INSTITUTE OF TECHNOLOGY UNIVERSITY OF CALIFORNIA MONTANA STATE UNIVERSITY UNIVERSITY OF NEVADA NEW MEXICO STATE UNIVERSITY OREGON STATE UNIVERSITY UNIVERSITY OF OREGON OSAKA UNIVERSITY UNIVERSITY OF SOUTHERN CALIFORNIA
STANFORD UNIVERSITY

UNIVERSITY OF TOKYO UNIVERSITY OF UTAH WASHINGTON STATE UNIVERSITY UNIVERSITY OF WASHINGTON AMERICAN MATHEMATICAL SOCIETY CHEVRON RESEARCH CORPORATION TRW SYSTEMS NAVAL WEAPONS CENTER 


\section{Pacific Journal of Mathematics}

\section{Vol. 25, No. $1 \quad$ September, 1968}

Glen Eugene Bredon, Cosheaves and homology................... 1

Robin Ward Chaney, A chain rule for the transformation of integrals in

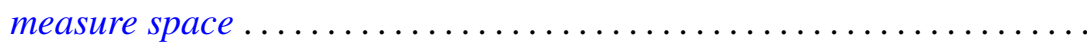

Colin W. Clark, On relatively bounded perturbations of ordinary differential operators................................... 59

John Edwin Diem, A radical for lattice-ordered rings.............. 71

Zeev Ditzian, On a class of convolution transforms ................ 83

Dennis Garoutte and Paul Adrian Nickel, A note on extremal properties characterizing weakly $\lambda$-valent principal functions............. 109

Shwu-Yeng Tzeng Lin, Fixed point properties and inverse limit spaces . . . 117

John S. Lowndes, Some dual series equations involving Laguerre polynomials ................................. 123

Kirti K. Oberai, Sum and product of commuting spectral operators ....... 129

J. N. Pandey and Armen H. Zemanian, Complex inversion for the generalized convolution transformation..................... 147

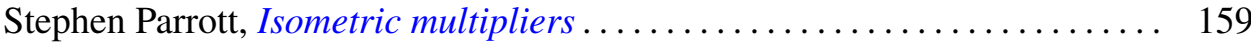

Manoranjan Prasad, Note on an extreme form .................. 167

Maciej Skwarczyński, A representation of a bounded function as infinite product in a domain with Bergman-Shilov boundary surface ......... 177

John C. Taylor, The Šilov boundary for a lattice-ordered semigroup ...... 185 Donald Reginald Traylor and James Newton Younglove, On normality and pointwise paracompactness ............................. 193

L. Tzafriri, Quasi-similarity for spectral operators on Banach spaces ... 